\title{
Quantum electrodynamics effects on NMR magnetic shielding constants of He-like and Be-like atomic systems
}

\author{
Carlos A. Gimenez, Karol Kozioł, and Gustavo A. Aucar* \\ Physics Department, Natural and Exact Science Faculty, Northeastern University of Argentina \\ and IMIT Institute of CONICET, Corrientes W3404AAS, Argentina
}

(Received 2 December 2014; revised manuscript received 12 January 2016; published 11 March 2016)

\begin{abstract}
NMR shielding constants for He- and Be-like atomic systems of $\mathrm{Ne}, \mathrm{Ar}, \mathrm{Kr}, \mathrm{Xe}$, and $\mathrm{Rn}$ have been calculated at the random-phase-approximation level of approach, including an estimation of QED corrections within the polarization propagator formalism. We show that QED effects enhance electron correlation when $Z$ becomes heavier, which happens with relativistic effects, and also that QED effects become smaller when going from more to less ionized systems. We studied two- and four-electron systems. Then such studies could easily be generalized to other many-electron systems. Results of calculations with our relatively simple model, which includes QED and electron correlation effects on the same theoretical grounds, have a summarized error in the range from $10 \%$ (for $\mathrm{Ne}$ ) up to $24 \%$ (for Rn), so that our accuracy is a little lower than for calculations on H-like systems. Our findings should stimulate the development and/or the application of more rigorous formalisms to get more accurate QED corrections to response properties in many-electron systems.
\end{abstract}

DOI: 10.1103/PhysRevA.93.032504

\section{INTRODUCTION}

How can we include many-electron QED contributions to response properties like NMR spectroscopic parameters, provided that exact analytic solutions do not exist? During the last couple of years few attempts were made to estimate the influence of QED effects on NMR nuclear magnetic shielding $\sigma$ of $\mathrm{H}$-like and He-like systems [1,2]. Theoretical treatments used in both references are fully relativistic, with that of Ref. [1] being based on the nonrelativistic QED expansion (only the zeroth-order approximation is obtained nonrelativistically). The difficulties are such that there are no actual calculations in the literature that give an estimate of such effects on $\sigma$ for many-electron systems.

The first theoretical models developed to introduce QED effects [the self-energy (SE) part] on $\sigma$ were published in 2002 [3,4]. In those models the main difficulties were related to solutions of formal expressions and implementations, like the integrals for the fourth level of the scattering matrix. More recently, Rudziński et al. published relativistic and QED corrections to $\sigma\left({ }^{3} \mathrm{He}\right)$ with an estimated precision of 0.1 part-per-billion (ppb) [1]. Its relativistic corrections were introduced through the Breit-Pauli Hamiltonian and were found to be $0.1 \%$ of the nonrelativistic one, with the QED corrections being $1 \%$ of the relativistic contributions. These findings are in line with previous suggestions [5]. Afterward, Yerokhin et al. [2] presented the results of ab initio calculations for several $\mathrm{H}$-like ions of $10 \leqslant Z \leqslant 92$, considering various QED contributions to $\sigma$, as well as Bohr-Weisskopf (BW) and quadrupole corrections. In Refs. [1,2] the first reliable results were given, but only for one- and two-electron atomic systems.

Few other general formalisms were developed to calculate QED effects on atomic and molecular properties. Most of them are still not implemented in computational codes $[4,6-8]$ or were implemented to calculate atomic properties like hyperfine and Zeeman splitting [9-12]. One of the main difficulties that

\footnotetext{
*gaaucar@conicet.gov.ar
}

they share is related to the way to handle the inclusion of the electron correlation.

There are several leading electronic effects, such as the electron correlation and relativistic effects, that should be included in order to get an accurate theoretical reproduction of the nuclear magnetic shielding. For heavy elements it is known that relativistic effects may be as large as the nonrelativistic (NR) ones [13]. When one looks for more accurate results, the effect of the nuclear size and QED corrections should be introduced. The BW corrections, meaning the effect induced by the spatial distribution of the nuclear magnetic moments, are of the same order of magnitude as the QED effects for H-like ions (for $Z>30$ ) [2].

As shown recently, electron correlation and relativistic effects are dependent on each other for NMR spectroscopic parameters [13]. Furthermore the nuclear charge distribution effects may be of the order of $9 \%$ for heavy-atom-containing molecules [14]. Then the next important issue to address for the calculation of $\sigma$ in many-electron systems is related to the likely mutual dependence of electron correlation and QED. How important are electron correlation effects when QED effects are properly considered? Or, vice versa, how large are the electron correlation effects in a QED framework? If they were independent of one another one could more easily figure out how to include both effects in a simpler calculation.

Quite recently, it was shown that the polarization propagator formalism can be derived from the path-integral formalism [8]. This fact gives new insights on how to include both effects together, through the consideration of the effects of external perturbations on a many-body quantum system that is described within a QED-based theoretical framework. Within the polarization propagator formalism the effect of an (external) perturbation can be described by using knowledge of the unperturbed, although correlated, many-body quantum system.

We shall show here how to get reliable QED corrections when including electron correlation up to first order (random phase approximation). There is no need to go one step further in the introduction of electron correlation, given that calculations 
at the RPA level of approach include most of these effects for the nuclear shielding in atomic systems.

\section{THEORETICAL MODEL}

We applied an effective model from which QED effects can be introduced in NMR shielding, following the ansatz suggested in Sec. 5 of Ref. [8]. We consider the ground state of an unperturbed atomic system described by means of a self-consistent-field (SCF) or multiconfiguration Dirac-Fock (MCDF) scheme which includes the leading QED effects. In addition to that we introduce the electron-correlation effects on response properties via perturbation theory.

\section{A. The NMR magnetic shielding}

Within the polarization propagator formalism, the $\sigma$ constant is written in terms of the so-called perturbators $\mathbf{b}$ and the principal propagator $\mathbf{P}$ as [13]

$$
\sigma=\mathbf{b}^{N} \mathbf{P}(E=0) \mathbf{b}^{B}+\text { c.c., }
$$

where $N$ corresponds to its nuclear magnetic moment index and $B$ is the external magnetic field. The principal propagator matrix at the RPA level of approach is $[15,16]$

$$
\mathbf{P}=\left(\begin{array}{ll}
\boldsymbol{A} & \boldsymbol{B}^{*} \\
\boldsymbol{B} & \boldsymbol{A}^{*}
\end{array}\right)^{-1} .
$$

Within the second quantization,

$$
\begin{aligned}
\boldsymbol{A}_{i a, j b} & =-\left\langle 0\left|\left[a_{i}^{\dagger} a_{a},\left[a_{b}^{\dagger} a_{j}, H_{0}\right]\right]\right| 0\right\rangle \\
& =\delta_{a b} \delta_{i j}\left(\varepsilon_{a}-\varepsilon_{i}\right)+\langle a j \| i b\rangle \\
& =A_{i a, j b}(0)+A_{i a, j b}(1)
\end{aligned}
$$

and

$$
\boldsymbol{B}_{i a, j b}=-\left\langle 0\left|\left[a_{i}^{\dagger} a_{a},\left[a_{j}^{\dagger} a_{b}, H_{0}\right]\right]\right| 0\right\rangle=\langle j i \| a b\rangle .
$$

Subindices $a, b, \ldots$ refer to unoccupied Dirac-Hartree-Fock (DHF) orbitals, and $i, j, \ldots$ stand for occupied DHF orbitals. The pure-zeroth-order approach (PZOA) is obtained when matrix elements of matrix $\mathbf{B}$ and all two-electron matrix elements of $\mathbf{A}$ are neglected [13]. In other words, only the matrix elements of $\mathbf{A}(0)$ are considered.

For NMR shielding the perturbators are written as

$$
\mathbf{b}_{i a}^{N}=\left\langle a\left|\frac{\boldsymbol{\alpha} \times \boldsymbol{r}_{N}}{r_{N}^{3}}\right| i\right\rangle, \quad \mathbf{b}_{j b}^{B}=\left\langle j\left|\boldsymbol{\alpha} \times \boldsymbol{r}_{G}\right| b\right\rangle,
$$

where $\mathbf{r}_{\mathbf{G}}=\mathbf{r}-\mathbf{R}_{G}$, with $\mathbf{R}_{G}$ being the gauge origin, and $\mathbf{r}_{N}=\mathbf{r}-\mathbf{R}_{N}$, with $\mathbf{R}_{N}$ being the nuclear origin. They are related by excitations from occupied to unoccupied orbitals. In the relativistic regime the set of unoccupied orbitals is split in two subsets, the positive and negative branches of energies. Excitations to negative- and positive-energy solutions are related by the diamagneticlike $\left(\sigma^{d}\right)$ and paramagneticlike $\left(\sigma^{p}\right)$ contributions to $\sigma$ [17].

Actual calculations are not performed using Eq. (1) but an algorithm that solves the product between the inverted principal propagator and one of the perturbators,

$$
\begin{aligned}
\sigma & =\mathbf{b}_{i a}^{N}\left(\mathbf{M}^{-1}\right)_{i a, j b} \mathbf{b}_{j b}^{B}+\text { c.c. } \\
& =\mathbf{b}_{i a}^{N} \mathbf{X}_{i a}^{\mathrm{RPA}(\mathrm{PZOA})} .
\end{aligned}
$$

The matrix $\mathbf{X}$ contains all the information related to the principal propagator and one of the two perturbators.

\section{B. Polarization propagators and the path-integral formalism}

As mentioned above, polarization propagators or doubletime Green's functions can be derived from the more general path-integral formalism [8]. This means that if one is able to define an adequate generating functional in an atomic or molecular orbital representation, one can get the polarization propagators from it. If the operators $V^{N}$ and $V^{B}$ are the ones that belongs to the perturbative Hamiltonian defining the NMR magnetic shielding [operators given in Eq. (5)], the adequate generating functional can be expressed as

$$
\begin{aligned}
Z_{\left[V^{N}, V^{B}\right]} & \left.=\int D \mid \tilde{\mathbf{h}}\right) e^{\mid \tilde{\mathbf{h}})\left(\mathbf{h}\left|E \hat{I}-\hat{H}_{0}\right| \tilde{\mathbf{h}}\right)\left(\mathbf{h} \mid+\left(V^{N} \mid \tilde{\mathbf{h}}\right)\left(\mathbf{h} \mid+\left(V^{B} \mid \tilde{\mathbf{h}}\right)(\mathbf{h} \mid\right.\right.} \\
& =Z_{\left[V^{N}=0, V^{B}=0\right]} e^{i W\left[V^{N}, V^{B}\right]},
\end{aligned}
$$

where

$$
W_{\left[V^{N}, V^{B}\right]}=\left(V^{N} \mid \tilde{\mathbf{h}}\right)\left(\mathbf{h}\left|E \hat{I}-\hat{H}_{0}\right| \tilde{\mathbf{h}}\right)^{-1}\left(\mathbf{h} \mid V^{B}\right)
$$

is the molecular orbital (MO) or atomic orbital (AO) representative of the Green's function corresponding to the quantum correlation between two perturbative interactions $\left(V^{N}\right.$ and $\left.V^{B}\right)$ applied on a molecular or atomic system. This functional $W$ is the generating functional of the Green's function that contains all connected Feynmann-like diagrams.

Equation (6) is easily obtained from Eq. (8). There are three terms. The first and the third ones are related to perturbators, and the second is the principal propagator. The $\boldsymbol{h}$ symbols stand for excitation operator manifolds [13].

Terms like $\left(V^{N} \mid \tilde{\boldsymbol{h}}\right)$ are binary products defined as

$$
\left(V^{N} \mid \tilde{\boldsymbol{h}}\right)=\left\langle 0\left|\left[V^{N \dagger}, \tilde{\boldsymbol{h}}\right]\right| 0\right\rangle .
$$

The ket $|0\rangle$ is the ground state of the unperturbed system.

\section{Electron correlation and QED effects}

As mentioned above, polarization propagators do permit us to consider the response of atomic and molecular systems to internal or external perturbations by applying perturbation theory at different levels of approaches. Then we will consider the unperturbed atomic system as described within a QED framework and include the electron correlation due to the interaction among orbital excitations as a perturbation. This is like considering the influence of potential $u(\phi)$ in Eq. (35) of Ref. [8].

In other words, $H_{0}$ is the unperturbed Hamiltonian of the $n$ electron atomic system that is described using the SCF procedure, and $H_{1}$ is the perturbative one-body Hamiltonian arising from the interaction among the nuclear spin and the external magnetic field, with the electronic framework of the atom.

Our proposal is to apply an expression equivalent to that of Eq. (6). We make then an ansatz that is in line with the one given in Ref. [8].

(i) Only leading QED corrections to both perturbators and principal propagators are enough to estimate an order of magnitude for QED corrections to shielding.

(ii) QED corrections on perturbators $\mathbf{b}^{B}$ will be vanishingly small. 
(iii) Matrix $\mathbf{X}$ will contain QED corrections at either level of approximation, RPA and PZOA.

Then we rewrite Eq. (6) to include QED effects within polarization propagator formalism at zeroth- and first-order levels of the approach:

$$
\sigma^{\mathrm{QED}}=\sum_{i, a} \mathbf{X}_{i a}^{\mathrm{QED}}\left[\mathbf{b}_{i a}^{N} C_{i a}\left(\frac{D C+\mathrm{QED}}{D C}\right)\right] .
$$

In this equation the matrix elements $\mathbf{b}_{i a}^{N}$ are scaled by coefficients $C_{i a}$. The procedure by which these matrix elements are estimated is one of the key points of this work.

\section{The coefficients $C\left(\frac{D C+\mathrm{QED}}{D C}\right)$}

In Eq. (10) the coefficients $C$ scale the matrix elements of perturbators to include QED effects on them. As shown in Eq. (5), when such matrix elements are built from atomic orbitals like $\left|n_{1} \kappa_{1} m_{1}\right\rangle$ and $\left|n_{2} \kappa_{2} m_{2}\right\rangle$, they can be written as

$$
\left\langle n_{1} \kappa_{1} m_{1}\left|\frac{(\boldsymbol{\alpha} \times \boldsymbol{r})}{r^{3}}\right| n_{2} \kappa_{2} m_{2}\right\rangle=A\left(\kappa_{1} m_{1} \kappa_{2} m_{2}\right) R^{(-2)}\left(n_{1} \kappa_{1} n_{2} \kappa_{2}\right)
$$

and

$$
\left\langle n_{1} \kappa_{1} m_{1}|(\boldsymbol{\alpha} \times \boldsymbol{r})| n_{2} \kappa_{2} m_{2}\right\rangle=A\left(\kappa_{1} m_{1} \kappa_{2} m_{2}\right) R^{(1)}\left(n_{1} \kappa_{1} n_{2} \kappa_{2}\right),
$$

where $A\left(\kappa_{1} m_{1} \kappa_{2} m_{2}\right)$ are some angular coefficients and $R^{(n)}$ are the radial integrals, defined as

$$
R^{(n)}\left(n_{1} \kappa_{1} n_{2} \kappa_{2}\right)=\int_{0}^{\infty} r^{n}\left(P_{n_{1} \kappa_{1}} Q_{n_{2} \kappa_{2}}+Q_{n_{1} \kappa_{1}} P_{n_{2} \kappa_{2}}\right) d r
$$

with $P_{n \kappa}$ and $Q_{n \kappa}$ being the radial parts of the one-electron wave function (Dirac bispinor):

$$
\begin{gathered}
\psi=\frac{1}{r}\left(\begin{array}{c}
P_{n, \kappa}(r) \Omega_{\kappa, j}^{m_{j}}(\theta, \phi) \\
i Q_{n, \kappa}(r) \Omega_{-\kappa, j}^{m_{j}}(\theta, \phi)
\end{array}\right) \\
C\left(\frac{D C+\mathrm{QED}}{D C}\right)_{i a}=\left\{\begin{array}{l}
(1+A)\left\{1+\left[C\left(\frac{V P}{D C}\right)-B\right]\right. \\
1
\end{array}\right.
\end{gathered}
$$

In order to quantify the QED influence on $\sigma$ we start by estimating the vacuum polarization (VP) effects on perturbators in Eq. (5). We need to define first the coefficients that will be used as scaling factors in Eq. (10). So

$$
\begin{aligned}
C_{1}\left(\frac{D C V}{D C}\right) & =\frac{\left[\left\langle n_{1} \kappa_{1} m_{1}\left|(\boldsymbol{\alpha} \times \boldsymbol{r})_{q}\right| n_{2} \kappa_{2} m_{2}\right\rangle\right]_{D C V}}{\left[\left\langle n_{1} \kappa_{1} m_{1}\left|(\boldsymbol{\alpha} \times \boldsymbol{r})_{q}\right| n_{2} \kappa_{2} m_{2}\right\rangle\right]_{D C}} \\
& =\frac{\left[R^{(1)}\left(n_{1} \kappa_{1} n_{2} \kappa_{2}\right)\right]_{D C V}}{\left[R^{(1)}\left(n_{1} \kappa_{1} n_{2} \kappa_{2}\right)\right]_{D C}}
\end{aligned}
$$

and

$$
\begin{aligned}
C_{2}\left(\frac{D C V}{D C}\right) & =\frac{\left(\left\langle n_{1} \kappa_{1} m_{1}\left|\frac{(\alpha \times \boldsymbol{r})_{q}}{r^{3}}\right| n_{2} \kappa_{2} m_{2}\right\rangle\right)_{D C V}}{\left(\left\langle n_{1} \kappa_{1} m_{1}\left|\frac{(\alpha \times r)_{q}}{r^{3}}\right| n_{2} \kappa_{2} m_{2}\right\rangle\right)_{D C}} \\
& =\frac{\left[R^{(-2)}\left(n_{1} \kappa_{1} n_{2} \kappa_{2}\right)\right]_{D C V}}{\left[R^{(-2)}\left(n_{1} \kappa_{1} n_{2} \kappa_{2}\right)\right]_{D C}}
\end{aligned}
$$

where indexes $D C$ and $D C V$ indicate wave functions calculated in a self-consistent manner by using the Dirac-Coulomb (-Breit) Hamiltonian and the Dirac-Coulomb(-Breit) Hamiltonian with the addition of the Uehling potential, respectively. We decided to introduce only one factor in this work because $C\left(\frac{D C V}{D C}\right) \simeq C\left(\frac{D C B V}{D C B}\right)$.

One can consider only one factor to introduce the VP effects on both perturbators:

$$
C\left(\frac{D C V}{D C}\right)=C_{1}\left(\frac{D C V}{D C}\right) C_{2}\left(\frac{D C V}{D C}\right),
$$

and then

$$
C\left(\frac{V P}{D C}\right)=C\left(\frac{D C V}{D C}\right)-1 .
$$

It is worth mentioning that $C\left(\frac{V P}{D C}\right)$ factors in each group of excitations, $n s-n^{\prime} s, n p_{1 / 2}-n^{\prime} p_{1 / 2}, n p_{3 / 2}-n^{\prime} p_{3 / 2}$, etc., depend weakly on $n^{\prime}$, so it is justified to use only one $C\left(\frac{V P}{D C}\right)$ factor within each group of orbitals.

Next, in order to estimate the influence of both QED corrections $(\mathrm{VP}+\mathrm{SE})$ we use the following factor: where $A=\left(\Delta \varepsilon_{i}^{V P}-\Delta \varepsilon_{a}^{V P}+\Delta \varepsilon_{i}^{S E}-\Delta \varepsilon_{a}^{S E}\right) /\left(\varepsilon_{i}^{D C}-\varepsilon_{a}^{D C}\right)$, $B=\left(\Delta \varepsilon_{i}^{V P}-\Delta \varepsilon_{a}^{V P}\right) /\left(\varepsilon_{i}^{D C}-\varepsilon_{a}^{D C}\right), \quad$ and $\quad D_{\mathrm{SE}}(Z \alpha)$, $D_{V P}(Z \alpha)$, and $D_{V P, p o}(Z \alpha)$ are coefficients taken from Yerokhin et al. [2,18].

Coefficients $D_{\mathrm{SE}}, D_{V P}$, and $D_{V P, p o}$ have been calculated for $\mathrm{H}$-like systems in such a way that the use of the $C\left(\frac{D C+\mathrm{QED}}{D C}\right)$ factor is justified only for $n s-n^{\prime} s$ excitations.

How do we obtain the $\mathrm{C}\left(\frac{D C+\mathrm{QED}}{D C}\right)$ coefficients in Eq. (10)? Let us use the following auxiliary symbols:

$$
M_{i a}=\langle i|(\boldsymbol{\alpha} \times \boldsymbol{r})| a\rangle\left\langle a\left|\frac{(\boldsymbol{\alpha} \times \boldsymbol{r})}{r^{3}}\right| i\right\rangle .
$$

$\Delta \varepsilon=\varepsilon_{i}^{D C}-\varepsilon_{a}^{D C}, \quad \Delta \varepsilon^{V P}=\Delta \varepsilon_{i}^{V P}-\Delta \varepsilon_{a}^{V P}, \quad \Delta \varepsilon^{S E}=\Delta \varepsilon_{i}^{S E}-$ $\Delta \varepsilon_{a}^{S E}$ for simplification. if $a$ is an electronic state, if $a$ is a positronic state,

Then using Eq. (6) at the PZOA level of approach,

$$
\begin{aligned}
& \sigma^{D C+V P} \\
& =\sum_{i, a} \frac{M_{i a}^{D C V}}{\Delta \varepsilon+\Delta \varepsilon^{V P}}=\sum_{i, a} \frac{M_{i a}^{D C}\left[1+C\left(\frac{V P}{D C}\right)\right]}{\Delta \varepsilon+\Delta \varepsilon^{V P}} \\
& =\sigma^{D C}+\Delta \sigma^{V P, p o}=\left(\sum_{i, a} \frac{M_{i a}^{D C}}{\Delta \varepsilon}\right)+\Delta \sigma^{V P, p o},
\end{aligned}
$$

where $\Delta \sigma^{V P, p o}$ is equivalent to the $\mathrm{VP}$ perturbed-orbital contribution taken from Ref. [18]. After short derivations,

$$
\Delta \sigma^{V P, p o}=\sum_{i, a} \frac{M_{i a}^{D C}\left[C\left(\frac{V P}{D C}\right)-\frac{\Delta \varepsilon^{V P}}{\Delta \varepsilon}\right]}{\Delta \varepsilon+\Delta \varepsilon^{V P}} .
$$


Next, we can write

$$
\begin{aligned}
\sigma^{D C+\mathrm{QED}} & =\sum_{i, a} \frac{M_{i a}^{D C} C\left(\frac{D C+\mathrm{QED}}{D C}\right)}{\Delta \varepsilon+\Delta \varepsilon^{V P}+\Delta \varepsilon^{S E}}=\sigma^{D C}+\Delta \sigma^{\mathrm{QED}} \\
& =\sigma^{D C}+\Delta \sigma^{V P, p o} \frac{\Delta \sigma^{\mathrm{QED}}}{\Delta \sigma^{V P, p o}} \\
& =\left(\sum_{i, a} \frac{M_{i a}^{D C}}{\Delta \varepsilon}\right)+\Delta \sigma^{V P, p o} \frac{D_{\mathrm{SE}}(Z \alpha)+D_{V P}(Z \alpha)}{D_{V P, p o}(Z \alpha)},
\end{aligned}
$$

where the expression

$$
\begin{aligned}
\Delta \sigma^{\mathrm{QED}} & =\Delta \sigma^{S E}+\Delta \sigma^{V P} \\
& =\alpha^{2}(Z \alpha)^{3} D_{\mathrm{SE}}(Z \alpha)+\alpha^{2}(Z \alpha)^{3} D_{V P}(Z \alpha)
\end{aligned}
$$

shows the QED influence on both electronic orbital properties and hyperfine interaction and the expression

$$
\Delta \sigma^{V P, p o}=\alpha^{2}(Z \alpha)^{3} D_{V P, p o}(Z \alpha)
$$

was introduced from Ref. [18]. Finally, linking Eqs. (21) and (22), we obtain the $C\left(\frac{D C+\mathrm{QED}}{D C}\right)$ coefficient in the form shown in Eq. (19).

\section{E. QED corrections within the MCDF approach}

The calculation of $C\left(\frac{D C V}{D C}\right)$ coefficients, the radial integrals defined in Eq. (13), and QED contributions to the orbital energies have been performed by means of the MCDFGME code $[9,19,20]$. This fully relativistic code is based on the well-established multiconfigurational Dirac-Fock approach.

For VP energy correction the Uehling potential [energy contribution term of order $\alpha(Z \alpha)^{4} m_{e} c^{2}$ [21]] has been included in a self-consistent manner, and the higher-order terms of less importance [Kallen and Sabry term [22] of order $\alpha^{2}(Z \alpha)^{4} m_{e} c^{2}$ and Blomqvist term [23] of order $\alpha(Z \alpha)^{6} m_{e} c^{2}$ ] have been included in a perturbational way.

The Uehling potential in the case of finite nuclear size and spherical symmetric nuclear charge distribution $\rho(\vec{r})$ can be expressed as [24]

$$
\begin{aligned}
U(\vec{r})= & -\frac{2}{3} \frac{\alpha \lambda_{e}}{r} \int_{0}^{\infty} d^{3} r^{\prime} r^{\prime} \rho\left(r^{\prime}\right) \\
& \times\left[K_{0}\left(\frac{2}{\lambda_{e}}\left|r-r^{\prime}\right|\right)-K_{0}\left(\frac{2}{\lambda_{e}}\left|r+r^{\prime}\right|\right)\right],
\end{aligned}
$$

where $\lambda_{e}$ is the Compton wavelength of the electron and the function $K_{0}(x)$ is defined as

$$
K_{0}(x)=\int_{1}^{\infty} d t e^{-x t}\left(\frac{1}{t^{3}}+\frac{1}{2 t^{5}}\right) \sqrt{t^{2}-1} .
$$

To estimate the SE contribution we used the Welton picture [25,26]. In this approach the self-energy correction for $s$-type Dirac-Fock orbitals is scaled from exact hydrogenic results by the following relation:

$$
\left(\Delta E_{n \kappa}\right)_{\mathrm{DF}}=\frac{\left\langle n \kappa\left|\nabla^{2} V_{\text {nucl }}(r)\right| n \kappa\right\rangle_{\mathrm{DF}}}{\left\langle n \kappa\left|\nabla^{2} V_{\text {nucl }}(r)\right| n \kappa\right\rangle_{\mathrm{Hyd}}}\left(\Delta E_{n \kappa}\right)_{\mathrm{Hyd}},
$$

where $V_{\text {nucl }}(r)$ is a nuclear potential. For one-electron systems the SE correction to the orbital energy has been calculated
TABLE I. Electron correlation corrections to relativistic $\sigma^{p}\left(\sigma^{t}\right)$ for neutral systems. ${ }^{\text {a }}$

\begin{tabular}{lccc}
\hline \hline Atom & RPA & PZOA & Difference \\
\hline $\mathrm{Ne}$ & $13.26(444.86)$ & $10.03(441.68)$ & $3.23(3.18)$ \\
$\mathrm{Ar}$ & $66.71(956.82)$ & $62.80(953.09)$ & $3.92(3.74)$ \\
$\mathrm{Kr}$ & $557.89(3229.48)$ & $537.58(3210.11)$ & $20.31(9.37)$ \\
$\mathrm{Xe}$ & $2023.13(6554.02)$ & $1963.21(6496.07)$ & $59.92(57.94)$ \\
$\mathrm{Rn}$ & $10937.48(19230.16)$ & $10548.88(18846.17)$ & $388.60(384.00)$ \\
\hline \hline
\end{tabular}

${ }^{\mathrm{a}}$ Basis sets used: cc-pVTZ for $\mathrm{Ne}$ and $\mathrm{Kr}$ and Dyall.cV3Z for $\mathrm{Xe}$ and Rn.

exactly by Mohr [27-29] and expressed as

$$
\Delta E_{n \kappa}=\frac{\alpha}{\pi} \frac{(Z \alpha)^{4}}{n^{3}} F_{n \kappa}(Z \alpha) m_{e} c^{2},
$$

where $F_{n \kappa}(Z \alpha)$ is a slowly varying function of $Z \alpha$.

It is worth emphasizing here that the contributions $\Delta \varepsilon^{S E}$ were evaluated approximately based on exact hydrogenic results and some more or less sophisticated scaling models (see, e.g., Refs. [5,30]).

\section{RESULTS AND DISCUSSION}

The calculation of $\sigma$ at the RPA level, both with QED and without QED corrections, has been performed by means of the DIRAC code [31], employing an isotropic Gaussian model for the nuclei. The electron correlation at a second-order level of approach (SOPPA) was obtained by applying the DALTON code [32]. For Ne, Ar, and Kr the NR correlation-consistent polarized valence triple zeta (cc-pVTZ) basis set was used [33-35], and the relativistic Dyall correlated valence triple zeta (Dyall.cV3Z) basis was used for Xe and Rn [36]. The $\varepsilon^{\mathrm{QED}}$ correction to the energy was included only for excitations from $s$-type orbitals.

As observed in Table I the electron correlation effects at the RPA level of approach for relativistic calculations of $\sigma$ in neutral systems are $0.30 \%$ for $\mathrm{Kr}, 0.90 \%$ for $\mathrm{Xe}$, and $2.04 \%$ for $\mathrm{Rn}$.

In addition to that, the NR electron correlation effects added from RPA to the SOPPA level of approach are smaller than $0.13 \%$ and $0.07 \%$ for Xe and Rn, respectively (see Table II). Then one can assume that most of the electron correlation is included at the RPA level. Furthermore QED corrections to $\sigma^{p}$ at the RPA level for He-like systems are given in Table III, with such corrections to $\sigma^{d}$ being vanishingly small, so the QED corrections to $\sigma^{d}$ are not included here.

In Fig. 1 we show the percentage contributions to the relativistic value of $\sigma^{p}$ of a He-like Rn atom with no QED corrections. The excitation $1 s \rightarrow 2 s(1 s \rightarrow 3 s)$ contributes $\cong 25.4 \%$ ( $\cong 5.6 \%)$. The contributions of excitations to the

TABLE II. NR correlated values of $\sigma^{p}\left(\sigma^{t}\right)$ for neutral systems.

\begin{tabular}{lccc}
\hline \hline Atom & RPA & SOPPA & Difference \\
\hline Xe Dyall cV3z & $0.0(5642.38)$ & $-7.11(5641.88)$ & $-7.11(-7.61)$ \\
Rn Dyall cV3z & $0.0(10728.12)$ & $-7.68(10720.24)$ & $-7.68(-7.88)$
\end{tabular}


TABLE III. QED corrections at the RPA level to $\sigma^{p}$ for He-like systems. cV4Z stands for correlated valence quadruple zeta.

\begin{tabular}{lrrr}
\hline \hline System and basis & DC+QED & \multicolumn{1}{c}{ DC } & Difference \\
\hline Ne Dyall cV4Z & 10.8172 & 10.8180 & -0.0008 \\
Ar Dyall cV4Z & 58.6025 & 58.6186 & -0.0161 \\
Kr Dyall cV4Z & 468.5153 & 468.8796 & -0.3643 \\
Xe Dyall cV4Z & 1601.4545 & 1603.6116 & -2.1571 \\
Rn Dyall cV3Z & 8114.4900 & 8139.6360 & -25.1460 \\
\hline \hline
\end{tabular}

continuous states have numbers of 65 and larger. In Fig. 2 the equivalent percentage contributions of a Be-like $\mathrm{Rn}$ atom are given. In this case the $1 s \rightarrow 3 s(2 s \rightarrow 3 s)$ excitation contributes $\cong 4.7 \%$ ( $\cong 8.5 \%)$.

The comparison of the patterns of contributions to $\sigma^{p}$ in He-like and Be-like systems due to excitations starting from $1 s$ orbitals or $2 s$ orbitals shows few similarities and differences. In the case of He-like systems the contributions of the first excitation, meaning $1 s \rightarrow 2 s$, are the largest compared with the whole pattern of contributions that ends in the continuum. That is not the case for Be-like systems. Still for excitations starting in the $2 s$ orbital in Be-like systems the pattern is similar to that of excitations starting in the $1 s$ orbitals in Helike systems, which may indicate that this could happen for excitations starting on the highest occupied atomic orbital.

Excitations starting on the $1 s$ orbital in Be-like systems show the opposite behavior, meaning the first four excitations each contribute much less than any of the contributions of excitations to the continuum states. Furthermore, the percentage contributions of all the excitations to the continuum of ionized $\mathrm{Rn}$ are $64.13 \%$ for its He-like configuration and $77.56 \%$ for its Be-like configuration.

QED corrections to $X$-like $(X=\mathrm{He}, \mathrm{Be})$ atomic systems are shown in Fig. 3. QED corrections on top of correlated calculations are larger than the uncorrelated ones. In the case of Be-like Rn they get close to $0.16 \%$ at the RPA level and $0.07 \%$ at the PZOA level.

Do QED effects enhance or diminish the electron correlation effects? For Be-like systems the QED corrections at

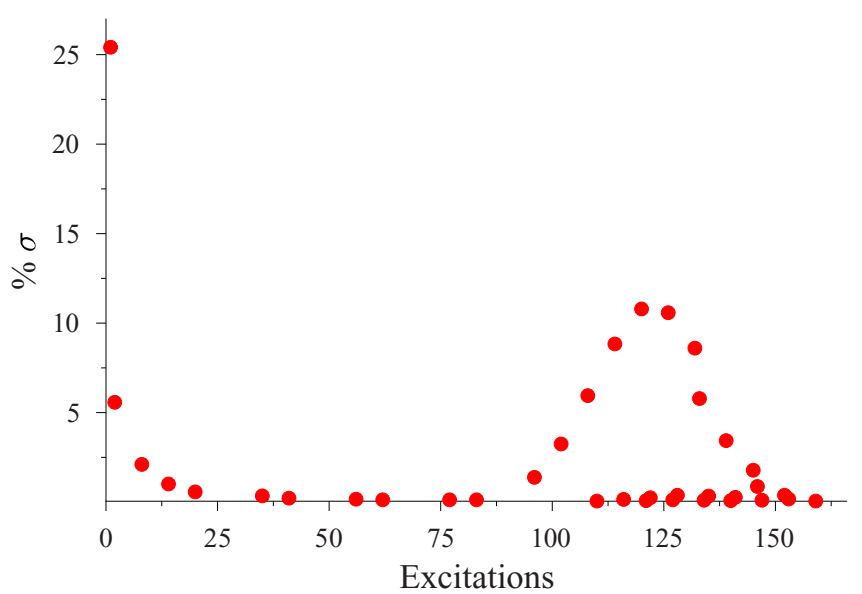

FIG. 1. Percentage contributions to $\sigma^{p}($ He-like Rn) due to excitations starting in $1 s$ orbitals.

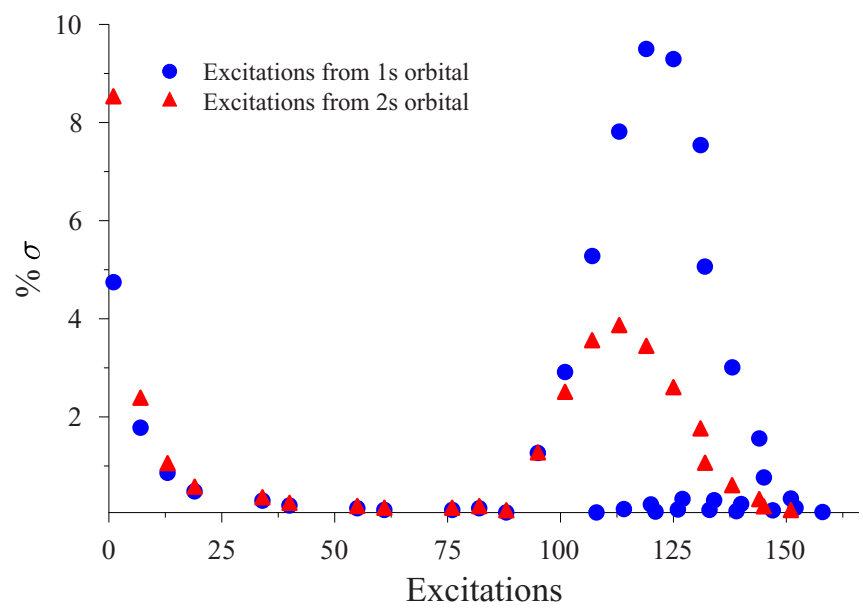

FIG. 2. Pattern of percentage contributions to $\sigma^{p}$ (Be-like Rn) due to excitations starting in $1 s$ and $2 s$ orbitals.

the RPA level are larger than the equivalent PZOA values. In Fig. 4 we show the percentage values of the calculation: [(RPA - PZOA) with QED - (RPA - PZOA) without QED]/[RPA - PZOA) without QED]. This shows that QED effects enhance electron correlation when $Z$ becomes heavier, although such an enhancement is larger for He- than for Be-like systems.

In order to estimate the errors of our numbers, a few points should be considered, according to Eq. (19).

First, the error of orbital energy values can be omitted because the precision of elemental constants used and the precision of numerical calculation are a couple of orders of magnitude larger than the precision of the numbers we present here. Then, the error originates from terms utilizing the $C\left(\frac{V P}{D C}\right)$ factor and from terms utilizing the $D_{\mathrm{SE}}$ and $D_{V P}$ factors. The uncertainty of the results from Yerokhin et al. [2,18] is the major source of error, and our values cannot have errors smaller than that. From those works the error of $D_{\mathrm{SE}}$ is in the range of about $0.02 \%-20 \%$ (see Table I in Ref. [18]), and the error of the QED contribution to $\sigma$ is about $8 \%-23 \%$ (see Table III in Ref. [18]). In this work there are no errors

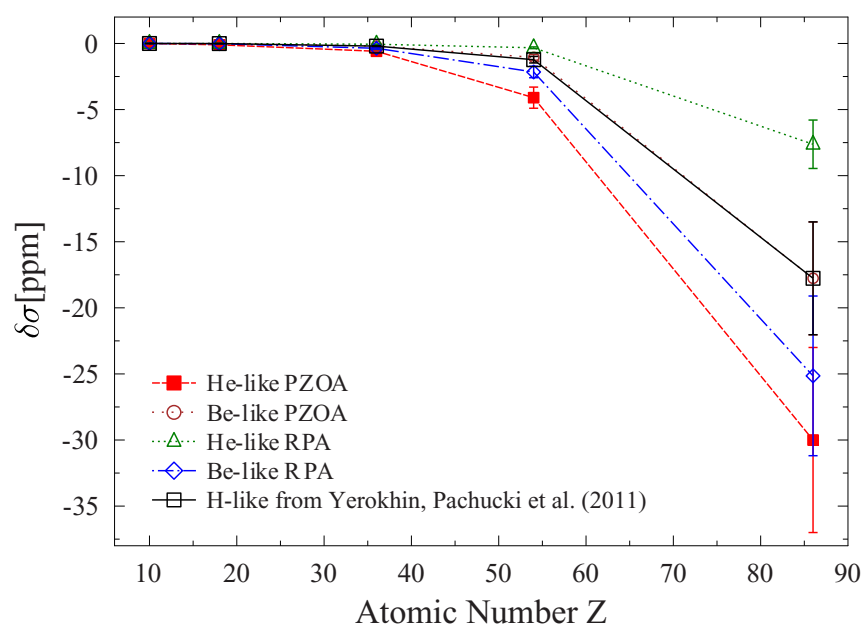

FIG. 3. QED corrections to $\sigma^{t}$ of He-like and Be-like atoms at RPA and PZOA levels of approach. 


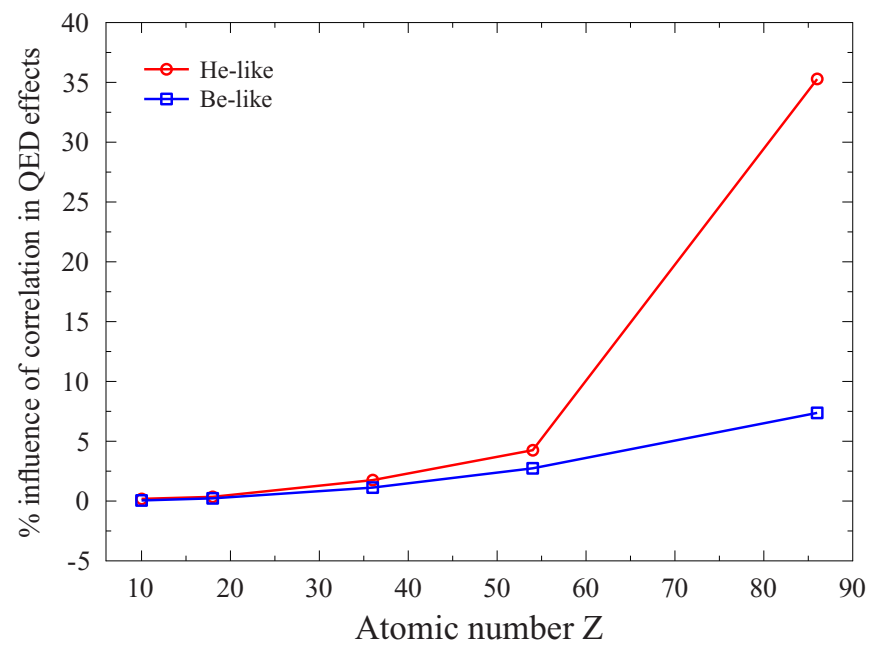

FIG. 4. Percentage enhancement of electron correlation due to the introduction of QED effects.

for $D_{V P}$ presented, but errors originating from the estimation of uncalculated vacuum-polarization diagrams is included in the total error of the QED contribution to $\sigma$. For the sake of estimation of errors of our numbers, we used percentage error values from Table III in Ref. [18] and interpolated (for $\mathrm{Ne}$ and $\mathrm{Xe}$ ) or extrapolated (for $\mathrm{Rn}$ ) them by using a quadratic polynomial. Another source of calculational errors, linked to the $C\left(\frac{V P}{D C}\right)$ factor, originated from the approximations used in our study. First, the difference between the $C\left(\frac{D C V}{D C}\right)$ and $C\left(\frac{D C B V}{D C B}\right)$ factors for a given $n s-n^{\prime} s$ pair is in the range of $0.3 \%-0.7 \%$ for the studied cases, which indicates the error of the $C\left(\frac{D C V}{D C}\right)=C\left(\frac{D C B V}{D C B}\right)$ approximation.

Second, the difference between the $C\left(\frac{V P}{D C}\right)$ factors for $n s-n^{\prime} s$ and $n s-n^{\prime \prime} s$ pairs is up to $5 \%$ (it is an upper-limit estimation based on MCDFGME calculations with good numerical convergence for excited states achieved); that indicates the error of using only one $C\left(\frac{V P}{D C}\right)$ factor within groups of $\left\{n s-n^{\prime} s, n s-n^{\prime \prime} s, \ldots\right\}$ orbitals.

Finally, our results may have a summarized error of $10.6 \%$, $12.6 \%, 16.8 \%, 20.1 \%$, and $24.0 \%$ for $\mathrm{Ne}, \mathrm{Ar}, \mathrm{Kr}, \mathrm{Xe}$, and $\mathrm{Rn}$, respectively, which mostly originated from the errors in the calculations of Yerokhin et al. [2,18].

\section{CONCLUSION}

The analysis of QED effects together with electron correlation on the response properties in many-electron atoms is still an almost unexplored area of research.

We proposed here an effective model, from which QED effects on NMR magnetic shielding of many-electron atomic systems can be estimated. This model is based on the ansatz suggested in Ref. [8], where the polarization propagator formalism was successfully derived from the path-integral formalism. We included the leading QED effects on both perturbators and the principal propagator, taking care of the fact that, within the polarization propagator formalism, one must consider the effect of external perturbations on unperturbed systems. QED effects were introduced in the perturbators by well-defined correcting factors. The uncorrected perturbators were calculated from the output of a multiconfiguration DiracFock scheme. In addition, the electron correlation effects were added at the RPA level of approach, which is good enough for atomic systems. Such electron correlations were calculated applying the same MCDF scheme.

Our results are based on state-of-the-art calculations of QED effects on H-like systems, which were accurately estimated from those of Refs. [2,18]. As a check of the accuracy of our numbers one should consider the closeness among our results and those published in Refs. [2,18].

What advance in both the estimate of the order of magnitude and dependence on QED and the electron correlation effects of the atomic NMR shielding did we introduce? Our results show that (a) QED corrections are larger when electron correlation is considered, (b) QED corrections for Be-like systems are smaller (absolute values) than for He-like systems, (c) QED effects enhance electron correlation when $Z$ becomes heavier, which happens with relativistic effects, and (d) QED effects become smaller when going from more to less ionized systems.

\section{ACKNOWLEDGMENTS}

We gratefully acknowledge support from the Argentinean Agency for the Promotion of Science and Technology (FONCYT PICT2012-1214), the Argentinean Council for Science and Technology (CONICET), and Gobierno de la Provincia de Corrientes, Argentina, which gave support for the short visit of K.K. to Corrientes.
[1] A. Rudziński, M. Puchalski, and K. Pachucki, J. Chem. Phys. 130, 244102 (2009).

[2] V. A. Yerokhin, K. Pachucki, Z. Harman, and C. H. Keitel, Phys. Rev. Lett. 107, 043004 (2011).

[3] R. H. Romero and G. A. Aucar, Int. J. Mol. Sci. 3, 914 (2002).

[4] R. H. Romero and G. A. Aucar, Phys. Rev. A 65, 053411 (2002).

[5] P. Pyykkö and L.-B. Zhao, J. Phys. B 36, 1469 (2003).

[6] W. Kutzelnigg, Chem. Phys. 395, 16 (2012).

[7] W. Liu and I. Lindgren, J. Chem. Phys. 139, 014108 (2013).

[8] G. A. Aucar, Phys. Chem. Chem. Phys. 16, 4420 (2014).

[9] P. Indelicato and J. Desclaux, MCDFGME, a multiconfiguration Dirac-Fock and general matrix elements program, release 2008, http://dirac.spectro.jussieu.fr/mcdf.
[10] B. J. McKenzie, I. P. Grant, and P. H. Norrington, Comput. Phys. Commun. 21, 233 (1980).

[11] V. M. Shabaev, Phys. Rep. 356, 119 (2002).

[12] A. V. Volotka, D. A. Glazov, V. M. Shabaev, I. I. Tupitsyn, and G. Plunien, Phys. Rev. Lett. 112, 253004 (2014).

[13] G. A. Aucar, R. H. Romero, and A. F. Maldonado, Int. Rev. Phys. Chem. 29, 1 (2010).

[14] A. F. Maldonado, C. A. Gimenez, and G. A. Aucar, J. Chem. Phys. 136, 224110 (2012).

[15] G. A. Aucar and J. Oddershede, Int. J. Quantum Chem. 47, 425 (1993).

[16] G. E. Scuseria, T. M. Henderson, and D. C. Sorensen, J. Chem. Phys. 129, 231101 (2008). 
[17] G. A. Aucar, T. Saue, L. Visscher, and H. J. A. Jensen, J. Chem. Phys. 110, 6208 (1999).

[18] V. A. Yerokhin, K. Pachucki, Z. Harman, and C. H. Keitel, Phys. Rev. A 85, 022512 (2012).

[19] J. Desclaux, Comput. Phys. Commun. 9, 31 (1975).

[20] J. Desclaux, Comput. Phys. Commun. 35, C-288 (1984).

[21] E. A. Uehling, Phys. Rev. 48, 55 (1935).

[22] G. Kallen and A. Sabry, K. Dan. Vidensk. Selsk. Mat. Fis. Medd. 29, 17 (1955).

[23] J. Blomqvist, Nucl. Phys. B 48, 95 (1972).

[24] L. W. Fullerton and G. A. Rinker, Phys. Rev. A 13, 1283 (1976).

[25] T. A. Welton, Phys. Rev. 74, 1157 (1948).

[26] P. Indelicato, O. Gorveix, and J. P. Desclaux, J. Phys. B 20, 651 (1987).

[27] P. J. Mohr, Phys. Rev. Lett. 34, 1050 (1975).
[28] P. J. Mohr and Y.-K. Kim, Phys. Rev. A 45, 2727 (1992).

[29] P. J. Mohr, Phys. Rev. A 26, 2338 (1982).

[30] J. A. Lowe, C. T. Chantler, and I. P. Grant, Radiat. Phys. Chem. 85, 118 (2013).

[31] L. Visscher, H. J. Aa. Jensen, R. Bast, and T. Saue, DIRAC, a relativistic $a b$ initio electronic structure program, release DIRAC13, 2013, http://www.diracprogram.org.

[32] DALTON, a molecular electronic structure program, release Dalton2015.X, 2015, http://daltonprogram.org.

[33] T. H. Dunning, Jr., J. Chem. Phys. 90, 1007 (1989).

[34] D. Woon and T. H. Dunning, Jr., J. Chem. Phys. 98, 1358 (1993).

[35] A. K. Wilson, D. Woon, K. Peterson, and T. H. Dunning, Jr., J. Chem. Phys. 110, 7667 (1999).

[36] K. Dyall, Theor. Chem. Acc. 117, 483 (2007). 